\title{
Invariant Sheaves
}

By

Masaki KASHIWARA *

\section{$\S 0 . \quad$ Introduction}

The sheaves of tangent vector fields, differential forms or differential operators are canonical. Namely they are invariant by the coordinate transformations. We call such sheaves invariant sheaves.

More precisely for a positive integer $n$, an invariant sheaf on $n$-manifold is given by the data: coherent $\mathcal{O}_{X}$-module $F_{X}$ for each smooth variety $X$ of dimension $n$ and an isomorphism $\beta(f): f^{*} F_{Y} \stackrel{\sim}{\rightarrow} F_{X}$ for any étale morphism $f: X \rightarrow Y$. We assume that $\beta(f)$ satisfies the chain condition (see $\S 1$ for the exact definition).

The purpose of this paper is to study the properties of invariant sheaves on $n$-manifold.

The first result is that the category $I(n)$ of invariant sheaves is equivalent to the category of modules over a certain group $G$ (with infinite dimension). Let us recall that the category of equivariant sheaves with respect to a transitive action is equivalent to the category of modules over the isotropy subgroup. In our case, manifold may be regarded as a homogeneous space of "the group" of all transformations, and the category of invariant sheaves is regarded as an equivariant sheaf with respect to this action. Let us take an $n$-dimensional vector space $V$ and let $G$ be the group of (formal) transformations that fix the origin. Hence $G$ is a semi-direct product of $G L_{n}$ and a projective limit of finitedimensional unipotent groups. This $G$ plays a role of the isotropy subgroup and we have

Theorem. The category of invariant sheaves are equivalent to the category of $G$-modules.

Received November 22, 1999.

1991 Mathematics Subject Classification(s): 14Axx

* Research Institute for Mathematical Sciences, Kyoto University, Sakyo-ku, Kyoto 6068502, Japan. 
The category $I(n)$ of invariant sheaves has other remarkable structure: filtered rigid tensor category. The group $G$ contains $G L(V)$ as a subgroup and it contains $\mathbb{G}_{\mathbf{m}}$ as its center. With respect to $\mathbb{G}_{\mathbf{m}}$, any $G$-module $M$ has a weight decomposition $M=\oplus M_{l}$. For any $l$ let us set $W_{l}(M)=\oplus_{l^{\prime} \leq l} M_{l^{\prime}}$. Then it turns out that $W_{l}(M)$ is a sub-G-module of $M$. Since the category of $G$-modules is equivalent to $I(n)$, any object $F$ of $I(n)$ has also a canonical finite filtration $W$, that we call the weight filtration. Thus, $I(n)$ has a structure of filtered category. We say that $F \in I(n)$ is pure of weight $w$ if $G r_{l}^{W} F=0$ for $l \neq w$. Then the category of pure invariant sheaves of weight $w$ is equivalent to the category of $G L(V)$-modules with weight $w$ (with respect to the $\mathbb{G}_{\mathbf{m}}$-action). Hence any pure invariant sheaf is semisimple.

Moreover $I(n)$ has a structure of tensor category by $\left(F_{1} \otimes F_{2}\right)_{X}=F_{1} \otimes_{\mathcal{O}_{X}}$ $F_{2}$. Thus $I(n)$ is a rigid tensor category.

The weight is preserved by the tensor product: $G r_{l}^{W}\left(F_{1} \otimes F_{2}\right)=\oplus_{l=l_{1}+l_{2}}$ $G r_{l_{1}}^{W}\left(F_{1}\right) \otimes G r_{l_{2}}^{W}\left(F_{2}\right)$. This structure is very similar to the category of mixed Hodge structures or motives. In particular, we can see easily

$$
\text { If } F_{\nu} \text { is pure of weight } w_{\nu} \quad(\nu=1,2) \text {, then }
$$

$$
\operatorname{Ext}^{\jmath}\left(F_{1}, F_{2}\right)=0 \quad \text { for } \quad w_{1}-w_{2}<j
$$

We conjecture

$$
\operatorname{Ext}^{\jmath}\left(F_{1}, F_{2}\right)=0 \quad \text { for } \quad j \neq w_{1}-w_{2} \quad \text { and } \quad j<n \text {. }
$$

This is translated to a conjecture of Lie algebra cohomology (Conjecture A.8 for Theorem A.3 in [F]. Hence (0.2) is already known for $2 j<n)$.

The group $\operatorname{Ext}^{1}\left(\mathcal{O}, \Omega^{1}\right)$ is one-dimensional, and its non-zero element is given by the extension $0 \rightarrow \Omega^{1} \rightarrow \Omega^{n \otimes-1} \otimes \mathcal{P}^{(1)}\left(\Omega^{n}\right) \rightarrow \mathcal{O} \rightarrow 0$. Here $\mathcal{P}^{(1)}\left(\Omega^{n}\right)_{X}$ $=p_{1 *}\left(\left(\mathcal{O}_{X \times X} / I^{2}\right) \otimes p_{2}^{*} \Omega_{X}^{n}\right)$ where $I$ is the defining ideal of the diagonal of $X \times X$, and $p_{1}$ and $p_{2}$ are the first and the second projection. Note that $\mathcal{O}$ has weight 0 and $\Omega^{1}$ has weight -1 . When $n=1, \operatorname{Ext}^{1}\left(\mathcal{O}, \Omega^{1 \otimes 2}\right)$ is non zero. Its non-zero element gives an extension

$$
0 \rightarrow \Omega^{1 \otimes 2} \stackrel{\varphi_{\rho}}{\rightarrow} K \stackrel{\varphi_{F}}{\rightarrow} \mathcal{O} \rightarrow 0
$$

This is connected with the Schwartzian derivative. Namely, if we take a coordinate $f$ of $X$ then the sequence (0.3) splits. Hence there is an element $s(f) \in K$ such that $\varphi_{1}(s(f))=0$. if we take another coordinate $g$, then there exists $\omega \in$ $\Omega_{X}^{1 \otimes 2}$ such that $\varphi_{0}(\omega)=s(g)-s(f)$. Then $\omega$ is given by $\{g ; f\}(d f)^{\otimes 2}$. Here $\{g ; f\}$ 
is the Schwartzian derivative $\left(d^{3} g / d^{3} f\right) /(d g / d f)-3\left(d^{2} f / d^{2} g\right)^{2} / 2(d f / d g)^{2}$. This explains the cocycle condition of the Schwartzian derivatives:

$$
\{h ; g\}(d g)^{\otimes 2}+\{g ; f\}(d f)^{\otimes 2}=\{h ; f\}(d f)^{\otimes 2} .
$$

For any $n$, the extension group $\oplus_{\jmath=0}^{n} \operatorname{Ext}^{\jmath}\left(\mathcal{O}, \Omega^{\jmath}\right)$ has a structure of ring by

$$
\begin{aligned}
\operatorname{Ext}^{\jmath}\left(\mathcal{O}, \Omega^{\jmath}\right) \otimes \operatorname{Ext}^{k}\left(\mathcal{O}, \Omega^{k}\right) & \rightarrow \operatorname{Ext}^{\jmath+k}\left(\mathcal{O}, \Omega^{\jmath} \otimes \Omega^{k}\right) \\
& \rightarrow \operatorname{Ext}^{\jmath+k}\left(\mathcal{O}, \Omega^{\jmath+k}\right) .
\end{aligned}
$$

There exists a canonical element $c_{\jmath} \in \operatorname{Ext}^{\jmath}\left(\mathcal{O}, \Omega^{\jmath}\right)$ such that

$$
\oplus \operatorname{Ext}^{\jmath}\left(\mathcal{O}, \Omega^{\jmath}\right) \simeq k\left[c_{1}, \cdots, c_{n}\right]^{\prime} .
$$

Here $k\left[c_{1}, \cdots, c_{n}\right]^{\prime}=k\left[c_{1}, \cdots, c_{n}\right] /\{$ degree $>n\}$. This follows from a theorem of Lie algebra cohomologies (cf.[F]). This $c_{\jmath}$ is connected with the Chern classes. Namely for any $n$-manifold $X$, we have the homomorphism

$$
\operatorname{Ext}_{I(n)}^{\jmath}\left(\mathcal{O}, \Omega^{\jmath}\right) \rightarrow \operatorname{Ext}_{\mathcal{O}_{\mathbf{I}}}^{\jmath}\left(\mathcal{O}_{X}, \Omega_{X}^{\jmath}\right)=H^{\jmath}\left(X ; \Omega_{X}^{\jmath}\right)
$$

and the image of $c_{\jmath}$ give the $j$-th Chern class of $X$.

\section{$\S 1 . \quad$ Definition}

We shall fix a positive integer $n$. Let $S$ be a scheme. Let us first define the category $\mathcal{S}_{n}(S)$ as follows. The objects of $\mathcal{S}_{n}(S)$ are smooth morphisms $X \stackrel{a}{\longrightarrow} T$ over $S$ with fiber dimension $n$. A morphism $\varphi$ from $X \stackrel{a}{\longrightarrow} T$ to $X^{\prime} \stackrel{a^{\prime}}{\longrightarrow} T^{\prime}$ in $\mathcal{S}_{n}(S)$ is a pair $\left(\varphi_{s}, \varphi_{b}\right)$ where $\varphi_{s}: X \rightarrow X^{\prime}, \varphi_{b}: T \rightarrow T^{\prime}$ are such that

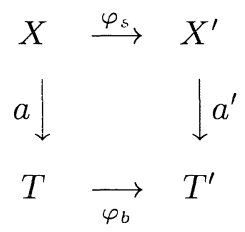

commutes and that $X \rightarrow X_{T^{\prime}}^{\times} T$ is an étale morphism.

An invariant sheaf $F$ is, by definition, given by following data:

(1.1) To any object $X \stackrel{a}{\longrightarrow} T$ in $\mathcal{S}_{n}(S)$,

assign a quasi-coherent $\mathcal{O}_{X}$-module $F(X \stackrel{a}{\longrightarrow} T)$,

(1.2) To any morphism $\varphi:(X \rightarrow T) \rightarrow\left(X^{\prime} \rightarrow T^{\prime}\right)$ in $\mathcal{S}_{n}(S)$,

assign an isomorphism $\beta(\varphi): \varphi_{s}^{*} F\left(X^{\prime} \rightarrow T^{\prime}\right) \stackrel{\sim}{\rightarrow} F(X \rightarrow T)$. 
We assume that these data satisfy the following associative law:

for a chain of morphisms $(X \rightarrow T) \stackrel{\varphi}{\longrightarrow}\left(X^{\prime}, T^{\prime}\right) \stackrel{\varphi^{\prime}}{\longrightarrow}\left(X^{\prime \prime} \rightarrow T^{\prime \prime}\right)$, the following diagram commutes

$$
\begin{array}{ccc}
\varphi_{s}^{*} \varphi_{s}^{*} F\left(X^{\prime \prime} \rightarrow T^{\prime \prime}\right) & \stackrel{\beta\left(\varphi^{\prime}\right)}{\longrightarrow} & \varphi_{s}^{*} F\left(X^{\prime} \rightarrow T^{\prime}\right) \\
\imath & \beta(\varphi) \downarrow \\
\left(\varphi_{s}^{\prime} \circ \varphi_{s}\right)^{*} F\left(X^{\prime \prime} \rightarrow T^{\prime \prime}\right) & \stackrel{\beta\left(\varphi^{\prime} \circ \varphi\right)}{\longrightarrow} & F(X \rightarrow T) .
\end{array}
$$

In the sequel for an object $X \stackrel{a}{\longrightarrow} T$ in $\mathcal{S}_{n}(S)$, we write $F_{X / T}$ for $F(X \rightarrow T)$ if there is no afraid of confusion.

The invariant sheaves form an additive category in an evident way. We denote this category by $I(n)_{S}$. If there is no afraid of confusion we denote it by $I(n)$.

The category $I(n)$ is a commutative tensor category. For objects $F_{1}$ and $F_{2}$ in $I(n), F_{1} \otimes F_{2}$ that associates $F_{1 X / T} \otimes_{\mathcal{O}_{X}} F_{2 X / T}$ for any objects $X \rightarrow T$ in $\mathcal{S}_{n}(S)$ is evidently an object of $I(n)$. Moreover $F_{1} \otimes F_{2} \cong F_{2} \otimes F_{1}$. Let us give several examples of invariant sheaves.

Example 1.1. The object $\mathcal{O} \in I(n)$. This associates to any $X \rightarrow T$ the sheaf $\mathcal{O}_{X}$.

Example 1.2. The object $\Omega^{k} \in I(n)$. This associates to any $X \rightarrow T$, the sheaf $\Omega_{X / T}^{k}$ of relative $k$-forms.

Example 1.3. The object $\Theta \in I(n)$. This associates to any $X \rightarrow T$ the sheaf $\Theta_{X / T}$ of relative tangent vectors.

Example 1.4. $S^{m}\left(\Omega^{k}\right)$. This associates $S^{m}\left(\Omega_{X / T}^{k}\right)$.

Example 1.5. For any object $X \rightarrow T$ in $\mathcal{S}_{n}(S)$, let $\Delta_{X / T}^{(m)}$ be the $m$-th infinitesimal neighborhood of the diagonal of $X \underset{T}{\times} X$. Namely if we denotes by $I$ the defining ideal of the diagonal $X \hookrightarrow X \underset{T}{\times} X$, then $\Delta_{X / T}^{(m)}$ is the subscheme of $X \underset{T}{\times} X$ defined by $I^{m+1}$. For $i=1,2$ let $p_{\imath}$ be the composition $\Delta_{X / T}^{(m)} \hookrightarrow$ $\underset{T}{\times} \underset{T}{\times} X \rightarrow X$ where the last arrow is the $i$-th projection. Then $\mathcal{P}^{(m)}$ associates 
$p_{1 *} \mathcal{O}_{\Delta_{X / T}^{(m)}}$. More generally, for any invariant sheaf $F, \mathcal{P}^{(m)}(F)$ that assigns $p_{1 *} p_{2}^{*} F_{X / T}$ is an invariant sheaf. Then there exists an exact sequence

$$
0 \rightarrow S^{m}\left(\Omega^{1}\right) \otimes F \rightarrow \mathcal{P}^{(m)}(F) \rightarrow \mathcal{P}^{(m-1)}(F) \rightarrow 0 .
$$

Example 1.6. $W_{m}(\mathcal{D})$. This associates the sheaf $W_{m}\left(\mathcal{D}_{X / T}\right)$ of the (relative) differential operators of order at most $m$. We regard this as an $\mathcal{O}_{X^{-}}$ module by the left multiplication.

Example 1.7. $W_{m}\left(\mathcal{D}^{o p}\right)$. This associates the same sheaf $W_{m}\left(\mathcal{D}_{X / T}\right)$ but we regard this as an $\mathcal{O}_{X}$-module by the right multiplication.

\section{§2. Finiteness and Flat Conditions}

\section{§2.1. Finiteness condition}

For the sake of simplicity, let us assume that

\section{$S$ is Noetherian.}

We keep this assumption in the rest of paper. An invariant sheaf $F$ is called coherent if $F_{X / T}$ is of locally finite type for any $X / T$ in $\mathcal{S}_{n}(S)$. Then $F_{X / T}$ is necessarily locally of finite presentation. In fact there exists locally in $X$ and $T$ a morphism $X / T$ to $\mathbf{A}^{n} \times S / S$ in $\mathcal{S}_{n}(S)$. Since $\mathbf{A}^{n} \times S$ is locally Noetherian, $F_{\mathbf{A}^{n} \times S / S}$ is a coherent $\mathcal{O}_{\mathbf{A}^{n} \times S}$-module. Hence the pull-back $F_{X / T}$ is locally of finite presentation.

Let us denote by $I_{c}(n)$ the full subcategory of $I(n)$ consisting of coherent invariant sheaves. Then we can see easily that $I_{c}(n)$ is an abelian category.

\section{§2.2. Flat condition}

An invariant sheaf $F$ is called invariant vector bundle if $F_{X / T}$ is flat over $T$ and locally of finite presentation over $\mathcal{O}_{X}$ for any $X / T$ in $\mathcal{S}_{n}(S)$.

Proposition 2.2.1. If $F$ is an invariant vector bundle then $F_{X / T}$ is locally free of finite rank for any $X / T$ in $\mathcal{S}_{n}(S)$. 
Proof. It is enough to show that $F_{\mathbf{A}^{n} \times S / S}$ is a locally free $\mathcal{O}_{\mathbf{A}^{n} \times S \text {-module. }}$ Since this is flat over $S$, it is enough to show that for any $s \in S, F_{\mathbf{A}^{n} \times s / s}$ is locally free. Thus we may assume that $S=\operatorname{Spec}(k)$ for a field $k$. Since $F_{\mathbb{A}^{n}}$ is equivariant over the translation group $G$ and $G$ acts transitively on $\mathbb{A}^{n}$. Hence $F$ is locally free.

Let us denote by $I^{b}(n)$ the category of invariant vector bundles. If $S$ is Spec $k$ for a field $k$, then $I^{b}(n)$ and $I_{c}(n)$ coincides. The functor $\otimes$ is an exact functor on $I^{b}(n)$, and a right exact functor on $I_{c}(n)$. For $F$ in $I^{b}(n)$, let $F^{*}$ be the invariant sheaf that associates $\mathcal{H o m}_{\mathcal{O}_{X}}\left(F_{X / T}, \mathcal{O}_{X}\right)$ with $X / T$ in $\mathcal{S}_{n}(S)$. With this, $I^{b}(n)$ has a structure of rigid tensor category.

\section{§3. Main Results}

\section{§3.1. Infinitesimal neighborhood}

Let $f: X \hookrightarrow Y$ be an embedding and let $I$ be the defining ideal of $f(X)$. Then for $m \geqq 0, \operatorname{Spec}\left(\mathcal{O}_{Y} / I^{m+1}\right)$ is called the $m$-th infinitesimal neighborhood of $X$ (or of $f: X \hookrightarrow Y$ ).

\section{§3.2. The group $G$}

Let us fix a locally free $\mathcal{O}_{S}$-module $\mathcal{V}$ of rank $n$, (e.g. $\left.\mathcal{V}=\mathcal{O}_{S}^{\oplus n}\right)$. Let $V$ be the associated vector bundle $\operatorname{Spec}\left(S_{\mathcal{O}_{S}}\left(\mathcal{V}^{*}\right)\right)$. Then $V \rightarrow S$ is an object of $\mathcal{S}_{n}(S)$. Let $i: S \rightarrow V$ be the zero section and let us denote by $W_{m}(V)$ its $m$-th infinitesimal neighborhood. Then $S=W_{0}(V) \subset W_{1}(V) \subset \cdots$ is an increasing sequence of subschemes of $V$. Let us set

$$
G(m)=\left\{g \in \operatorname{Aut}_{S}\left(W_{m}(V)\right) ; g \text { fixes } W_{0}(V)\right\} .
$$

Then $G(m)$ is an affine smooth group scheme over $S$ and we have a canonical smooth surjective morphism $G(m) \rightarrow G(m-1)$. Let $G$ be the projective limit of $\{G(m) ; m \in \mathbb{N}\}$. Then $G$ is an affine group scheme over $S$. Let $W^{m}(G)$ be the kernel of $G \rightarrow G(m)$. Then

$$
\begin{aligned}
& W^{0}(G)=G, \\
& G / W^{m}(G)=G(m), \\
& G / W^{1}(G)=G L(V) .
\end{aligned}
$$

For $m>0, W^{m}(G) / W^{m+1}(G)$ is an abelian unipotent group scheme corresponding $S^{m}\left(\mathcal{V}^{*}\right) \otimes \mathcal{V}$ (e.g. $\left.W^{m}(G) / W^{m+1}(G)=\operatorname{Spec}\left(S\left(\left(S^{m}\left(\mathcal{V}^{*}\right) \otimes \mathcal{V}\right)^{*}\right)\right)\right)$. Note that $G$ is a semi-direct product of $G L(V)$ and $W_{1}(G)$. 


\section{§3.3. Statement}

A $G$-module $M$ is by definition a quasi-coherent $\mathcal{O}_{S}$-module with a structure of $\pi_{*} \mathcal{O}_{G}$-comodule, where $\pi: G \rightarrow S$ is the canonical projection. A $G$-module $M$ is called coherent if it is coherent over $\mathcal{O}_{S}$.

If $M$ is a coherent $G$-module then the action of $G$ on $M$ comes from a $G(m)$-module structure on $M$ for $m \gg 0$. Our main result is the following.

Theorem 3.1. The category $I_{c}(n)$ of coherent invariant sheaves is equivalent to the category $\operatorname{Mod}_{c}(G)$ of coherent $G$-modules.

Remark. Let $X \rightarrow S$ be a smooth morphism of fiber dimension $n$ and let $i: S \rightarrow X$ be its section. Let $W_{m}(i)$ be the $m$-th infinitesimal neighborhood of $i$. Let $G(m)_{2}$ be the group of automorphisms of $W_{m}(i)$ that fix $W_{0}(i)=i(S)$. Then $G_{\imath}=\underset{m}{\lim _{m}} G(m)_{\imath}$ is isomorphic to $G$ locally in $S$ with respect to the Zariski topology. Moreover the category of $G$-modules is equivalent to the category of $G_{2}$-modules.

\section{§4. The Weight Filtration}

\section{§4.1. Definition}

The group $G$ contains $\mathbf{G}_{\mathbf{m}}$ as the homothetie subgroup by $\mathbf{G}_{\mathbf{m}} \times V \ni$ $(t, x) \mapsto t x \in V$. Any coherent $G$-module $M$ has a weight decomposition

$$
M=\underset{\ell \in \mathbf{Z}}{\oplus} M_{\ell}
$$

Here $\mathbf{G}_{\mathbf{m}}$ acts on $M_{\ell}$ by

$$
t u=t^{\ell} u \quad \text { for } \quad u \in M_{\ell}, t \in \mathbf{G}_{\mathbf{m}} .
$$

We set

$$
W_{\ell}(M)=\underset{m \leq \ell}{\oplus} M_{m}
$$

We call this the weight filtration of $M$.

\section{§4.2. Weight filtration}

We shall prove that $W_{\ell}(M)$ is a sub-G-module of $M$. We shall embed $\mathbf{G}_{\mathbf{m}}$ into $\mathbb{A}^{1}$. Let $\mathbb{G}_{\mathbf{m}} \times G \stackrel{\varphi}{\longrightarrow} G$ be the modified adjoint action $\varphi(t, g)=t^{-1} g t$. We can see easily the following lemmas. 
Lemma 4.2.1. $\varphi: \mathbb{G}_{\mathbf{m}} \times G \rightarrow G$ extends uniquely to a morphism $\tilde{\varphi}:$ $\mathbf{A}^{1} \times G \rightarrow G$.

Lemma 4.2.2. For any $\ell \geq 0, \tilde{\varphi}: \mathbb{A}^{1} \times W^{\ell}(G) \rightarrow W^{\ell}(G)$ is equal to the second projection modulo $t^{\ell}$, i.e. the composition $W_{\ell-1}\left(\mathbb{A}^{1}\right) \times W^{\ell}(G) \rightarrow$ $\mathbb{A}^{1} \times W^{\ell}(G) \rightarrow W^{\ell}(G)$ equals the second projection. Here $W_{\ell-1}\left(\mathbb{A}^{1}\right)=$ $\operatorname{Spec}\left(\mathbb{Z}[t] / t^{\ell} \mathbf{Z}[t]\right)$.

These lemmas imply the following result.

Proposition 4.2.3. Let $M$ be a $G$-module.

(i) $W_{\ell}(M)$ is a sub-G-module.

(ii) For $g \in W^{m}(G),(g-1)$ sends $W_{\ell}(M)$ into $W_{\ell-m}(G)$.

Here $g \in W^{m}(G)$ means $g \in \operatorname{Hom}_{S}\left(T, W^{m}(G)\right)$ for an $S$-scheme $T$. In the sequel, we use the similar abbreviation.

Proof. For any $g \in G, b \in \mathbb{Z}$ and $u_{b} \in M_{b}$ let us write $g u_{b}=\Sigma g_{a b} u_{b}$ with $g_{a b} u_{b} \in M_{a}$. Then $\varphi(t, g) u_{b}=\Sigma t^{b-a} g_{a b} u_{b}$. Since this is a polynomial in $t, g_{a b} u_{b}=0$ for $a>b$. This implies (i). If $g \in W^{m}(G)$, then the coefficients of $t^{c}$ in $\Sigma t^{b-a} g_{a b} u_{b}(0<c<m)$ vanishes. Hence $g_{a b} u_{b}=0$ for $b>a>b-m$. Thus $g u_{b}-u_{b} \in \underset{a \leq b-m}{\oplus} M_{a}$. This shows (ii).

Since $M$ is coherent, $W(M)$ is a finite filtration of $M$. For $a, b \in \mathbb{Z}$ with $a \leq b$, we say that $M$ has weights in $[a, b]$ if $W_{b}(M)=M$ and $W_{a-1}(M)=0$. For $w \in \mathbb{Z}$, we say that $M$ is pure of weight $w$ if $M$ has weights in $[w, w]$.

Corollary 4.2.4. If $M$ has weights in $[a, b]$, then the $G$-module structure of $M$ comes from a unique $G(b-a)$-module structure on $M$.

\section{$\S 5$. Functor $\Phi$}

\section{§5.1. Definition}

Let $F$ be a coherent invariant sheaf in $I_{c}(n)$. Let $i: S \rightarrow V$ be the zero section of the vector bundle $V \rightarrow S$ (c.f. $\S 3$ ). Set $\Phi(F)=i^{*} F_{\mathrm{I}^{\prime} S}$. Then $\Phi(F)$ is a coherent $\mathcal{O}_{S}$-module. In the sequel we shall endow a $G$-module structure on $\Phi(F)$. 


\section{§5.2. Weight decomposition}

The group $G L(V)$ acts on $V$ and hence on $i^{*} F_{V / S}$. Therefore $\Phi(F)$ is evidently a $G L(V)$-module. Since $\mathbf{G}_{\mathbf{m}}$ is contained in $G L(V)$ as the center, $\Phi(F)$ has a weight decomposition

$$
\Phi(F)=\underset{l \in \mathbb{Z}}{\oplus} \Phi(F)_{l}
$$

where $t \in \mathbf{G}_{\mathbf{m}}$ acts on $\Phi(F)_{l}$ by $t^{l}$.

As in $\S 4$, we set

$$
W_{l}(\Phi(F))=\underset{l^{\prime} \leq l}{\oplus} \Phi(F)_{l^{\prime}}
$$

Then $W$ is a finite filtration on $\Phi(F)$. We call it the weight filtration of $\Phi(F)$.

Similarly to the $G$-module case, we say that for $a \leq b, F$ is with weight in $[a, b]$ if $W_{b}(\Phi(F))=\Phi(F)$ and $W_{a-1}(\Phi(F))=0$.

Let $X \rightarrow T$ be an object in $\mathcal{S}_{n}(S)$ and $i: T \rightarrow X$ its section.

Proposition 5.2.1. Let $f$ and $g$ be morphisms in $\mathcal{S}_{n}(S)$ from $X \rightarrow T$ to $X^{\prime} \rightarrow T^{\prime}$. Let $i: T \rightarrow X$ be a section and let $T^{(m)}$ be its $m$-th infinitesimal neighborhood.

Let $F$ be a coherent invariant sheaf with weights in $[a, b]$. We assume

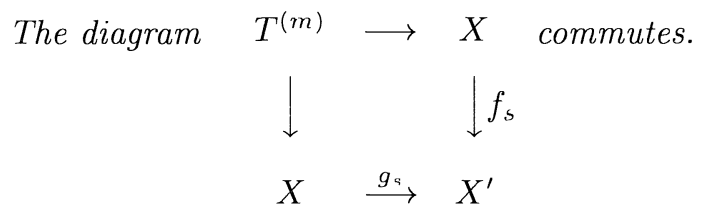

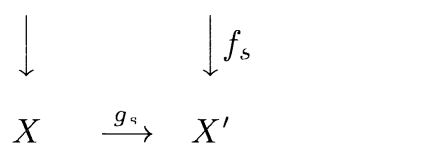

Then the following diagram commutes:

$$
\begin{array}{ccc}
\left(f_{s} \circ i\right)^{*} F_{X^{\prime} / T^{\prime}} & =i^{*} f_{s}^{*} F_{X^{\prime} / T^{\prime}} & \searrow^{\beta(f)} \\
\downarrow & \nearrow_{\beta(g)} & i^{*} F_{X / T} . \\
\left(g_{s} \circ i\right)^{*} F_{X^{\prime} / T^{\prime}}=i^{*} g_{s}^{*} F_{X^{\prime} / T^{\prime}} &
\end{array}
$$

The proof will be given in $\S 5.4$.

Admitting this proposition for a while, we shall give its corollary. 
Let $T$ be an $S$-scheme and $T^{(m)}$ a $T$-scheme. We assume that locally in $T, T^{(m)}$ is isomorphic to the $m$-th infinitesimal neighborhood of a section $T \rightarrow X$ of a smooth $T$-scheme $X \rightarrow T$ with fiber dimension $n$.

Corollary 5.2.2. Let $F$ be a coherent invariant sheaf with weights in

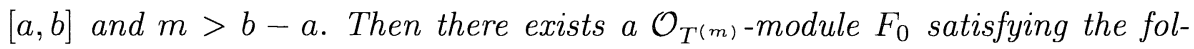
lowing properties (5.2.5) and (5.2.6).

(5.2.5) For $g: T^{\prime} \rightarrow T$, let $X^{\prime} \rightarrow T^{\prime}$ be an object of $\mathcal{S}_{n}(S)$ and let $j^{\prime}: T^{\prime(m)}=$ $T^{\prime} \times T^{(m)} \hookrightarrow X^{\prime}$ be an embedding by which $T^{(m)}$ is the $m$-th infinitesimal neighborhood of $i^{\prime}: T^{\prime} \hookrightarrow T^{\prime(m)} \hookrightarrow X^{\prime}$. Then there is an isomorphism $\gamma\left(j^{\prime}\right)$ : $i^{\prime *} F_{X^{\prime} / T^{\prime}} \stackrel{\sim}{\longrightarrow} g^{*} F_{0}$.

(5.2.6) $\gamma\left(j^{\prime}\right)$ satisfies the chain condition. Namely let $f:\left(X^{\prime \prime} \rightarrow T^{\prime \prime}\right) \rightarrow$ $\left(X^{\prime} \rightarrow T^{\prime}\right)$ be a morphism in $\mathcal{S}_{n}(S), j^{\prime \prime}: T^{\prime \prime} \underset{T}{\times} T^{(m)} \hookrightarrow X^{\prime \prime}$ a morphism over $j^{\prime}$ and $i^{\prime \prime}$ the composition of $T^{\prime \prime} \hookrightarrow T_{T}^{\prime \prime} \underset{T}{\times} T^{m}$ and $j^{\prime \prime}$. Then the diagram

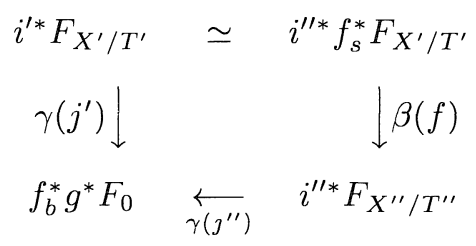

commutes.

Since the proof is straightforward we omit the proof.

\section{§5.3. Deformation of Normal cone}

In order to prove Proposition 5.2.1, we use the deformation of normal cone. Let us recall its definition. Let $X$ be a scheme and $Y \subset X$ a subscheme defined by an ideal $I$.

Let $t$ be an indeterminate and consider the ring

$$
\bigoplus_{n \in \mathbb{Z}} I^{n} t^{-n} \subset \mathcal{O}_{X}\left[t, t^{-1}\right]
$$

Here we understand $I^{n}=\mathcal{O}_{X}$ for $n \leq 0$.

Set $\tilde{C}_{Y / X}=\operatorname{Spec}\left(\oplus I^{n} t^{-n}\right)$ and let $q: \tilde{C}_{Y / X} \rightarrow X$ be the projection. This is called the deformation of normal cone. Then $t$ gives a morphism $\tilde{C}_{Y / X} \rightarrow \mathbb{A}^{1}$. 
Then $p^{-1}(0)$ is isomorphic to the normal cone $N_{Y / X}=\operatorname{Spec}\left(\underset{n \geq 0}{\oplus} I^{n} / I^{n+1}\right)$ and $p^{-1}\left(\mathbf{A}^{1} \backslash\{0\}\right) \sim X \times\left(\mathbb{A}^{1} \backslash\{0\}\right)$. The homomorphism $\underset{n}{\oplus} I^{n} t^{-n} \rightarrow$ $\underset{n \geq 0}{\oplus} \mathcal{O}_{X} t^{n} \rightarrow \underset{n \geq 0}{\oplus} \mathcal{O}_{Y} t^{n}$ gives the embedding $Y \times \mathbf{A}^{1} \subset \tilde{C}_{Y / X}$

If $X$ and $Y$ are smooth over $T$, then $\tilde{C}_{Y / X}$ is also smooth over $T$. If there is a smooth morphism $X^{\prime} \stackrel{f}{\longrightarrow} X$ and $f^{-1} Y \cong Y^{\prime}$, then there is a Cartesian diagram

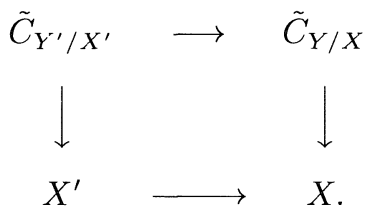

If $X$ is a vector bundle over $T$ and if $Y$ is the zero section of $X \rightarrow T$, then there is a unique isomorphism $X \times \mathbf{A}^{1} \stackrel{\sim}{\longrightarrow} \tilde{C}_{Y / X}$ such that $X \times \mathbf{A}^{1} \stackrel{\sim}{\longrightarrow} \tilde{C}_{Y / X} \rightarrow X$ is given $(x, t) \rightarrow t x$ and $X \times \mathbf{A}^{1} \cong \tilde{C}_{Y / X} \stackrel{p}{\longrightarrow} \mathbf{A}^{1}$ is the second projection.

\section{§5.4. Proof of Proposition 5.2.1}

Let us prove Proposition 5.2.1. By [EGA], we may assume $T$ to be Noetherian. By replacing $T$ with $S$ we may assume $T=S$. Locally in $Y$, there exists a morphism from $Y \rightarrow S$ to $V \rightarrow S$ in $\mathcal{S}_{n}(S)$ such that the composition $S \rightarrow X \rightarrow Y \rightarrow V$ coincides with the zero section. Hence replacing $Y \rightarrow S$ with $V \rightarrow S$ we may assume from the beginning that

$$
\begin{aligned}
& Y=V \\
& S \rightarrow X \rightarrow Y \text { coincides with the zero section. }
\end{aligned}
$$

Hence $\tilde{C}_{S / Y} \cong Y \times \mathbf{A}^{1}$ as seen in the preceding section. Thus we obtain a diagram of schemes over $S \times \mathbf{A}^{1}$.

$$
\begin{aligned}
& \tilde{C}_{S / X} \longrightarrow X \times \mathbf{A}^{1} \\
& \tilde{f}_{s} \llbracket \tilde{g}_{s} \quad f_{s} \times \imath d \llbracket g_{s} \times \imath d \\
& Y \times \mathbf{A}^{1} \cong \tilde{C}_{S / Y^{\prime}} \longrightarrow Y \times \mathbf{A}^{1}
\end{aligned}
$$

Note that $\tilde{f}_{s}$ and $\tilde{g}_{s}$ are étale and hence $\tilde{f}_{s}$ and $\tilde{g}_{s}$ give morphisms $\tilde{f}$ and $\tilde{g}$ from $\left(\tilde{C}_{S / X} \rightarrow S \times \mathbf{A}^{1}\right)$ to $\left(\tilde{C}_{S / Y} \rightarrow S \times \mathbf{A}^{1}\right)$ in $\mathcal{S}_{n}(S)$. 
Lemma 5.4.1. $\quad \tilde{f}_{s}$ and $\tilde{g}_{s}$ are equal modulo $t^{m}$, i.e.

$$
\operatorname{Spec}\left(\mathcal{O}_{\tilde{C}_{S / X}} / t^{m} \mathcal{O}_{\tilde{C}_{S / X}}\right) \rightarrow \tilde{C}_{S / X} \underset{\tilde{g}_{s}}{\stackrel{\tilde{f}_{s}}{\longrightarrow}} \tilde{C}_{S / Y}
$$

commutes (i.e. the two possible compositions are equal).

Proof. Let $I_{X} \subset \mathcal{O}_{X}$ and $I_{Y} \subset \mathcal{O}_{Y}$ be the defining ideal of $S \subset X$ and $S \subset Y$. Then by (5.2.3), $\mathcal{O}_{Y} \underset{g^{*}}{\stackrel{f^{*}}{\longrightarrow}} \mathcal{O}_{X} \rightarrow \mathcal{O}_{X} / I_{X}^{1+m}$ commutes. Hence $I_{Y} \underset{g^{*}}{\stackrel{f^{*}}{\longrightarrow}} I_{X} \rightarrow I_{X} / I_{X}^{1+m}$ commutes. Thus $I_{Y}^{l} \underset{g^{*}}{\stackrel{f^{*}}{\longrightarrow}} I_{X}^{l} \rightarrow I_{X}^{l} / I_{X}^{l+m}$ commutes for $l \geq 1$.

Hence $\bigoplus_{l} I_{Y} t^{-l} \stackrel{f^{+}}{\underset{g^{*}}{\rightrightarrows}} \oplus_{l} I_{X}^{l} t^{-l} \rightarrow \mathcal{O}_{\tilde{C}_{S / X}} / t \mathcal{O}_{\tilde{C}_{S / X}}=\underset{0 \leq l \leq m}{\oplus}\left(\mathcal{O}_{X} / I_{x}^{l}\right) t^{m-l} \oplus$ $\underset{l \geq 1}{\oplus}\left(I_{X}^{l} / I_{X}^{l+m}\right) t^{-l}$ commutes.

Now let $\tilde{j}: S \times \mathbb{A}^{1} \rightarrow \tilde{C}_{S / X}$ be the canonical embedding. Let $\tilde{j}_{Y}$ be the composition $\tilde{f}_{s} \circ \tilde{j}=\tilde{g}_{s} \circ \tilde{j}$.

Then we obtain the homomorphism $\tilde{\varphi}$ :

$$
\begin{aligned}
& \tilde{j}_{Y}^{*} F\left(Y \times \mathbf{A}^{1} \rightarrow S \times \mathbf{A}^{1}\right) \rightarrow \tilde{j}_{Y}^{*} F\left(\tilde{C}_{S / Y} \rightarrow S \times \mathbb{A}^{1}\right) \\
& \cong \tilde{j}^{*} \tilde{f}_{S}^{*} F\left(\tilde{C}_{S / Y} \rightarrow S \times \mathbf{A}^{1}\right) \stackrel{\beta(\tilde{f})}{\longrightarrow} \tilde{f}^{*} F\left(\tilde{C}_{S / X} \rightarrow S \times \mathbb{A}^{1}\right) \\
& \stackrel{\sim}{\sim} \tilde{j}^{*} \tilde{g}_{s}^{*} F\left(\tilde{C}_{S / Y} \rightarrow S \times \mathbf{A}^{1}\right) \cong \tilde{j}_{Y}^{*} F\left(\tilde{C}_{S / Y} \rightarrow S \times \mathbb{A}^{1}\right) \\
& \stackrel{\beta(\tilde{g})}{\sim} j_{Y}^{*} F\left(Y \times \mathbf{A}^{1} \rightarrow S \times \mathbb{A}^{1}\right) .
\end{aligned}
$$

Let us denote by $\varphi$ the composition

$$
\Phi(F) \simeq j^{*} F_{Y} \stackrel{\sim}{\sim} j^{*} f^{*} F_{Y} \stackrel{\beta(F)}{\longrightarrow} j^{*} F_{X} \underset{\beta(g)}{\stackrel{\sim}{\sim}} j^{*} g^{*} F_{Y} \sim i^{*} F_{Y} \sim \Phi(F) .
$$

Then outside $t \neq 0, \tilde{\varphi}$ coinsides with $t^{-1} \varphi t$. Thus $t^{-1} \varphi t$ extends to $t=0$, and equals to the identity modulo $t^{m}$ by Lemma 5.4.1. Now let us write

$$
\begin{gathered}
\varphi(u)=\sum_{\nu} \varphi_{\nu \mu}(u) \quad \text { for } u \in \Phi(F)_{\mu} \\
\text { with } \varphi_{\nu \mu}(u) \in \Phi(F)_{\nu} .
\end{gathered}
$$

Then $\tilde{\varphi}(u)=\sum t^{-\nu} \varphi_{\nu \mu}(t u)=\sum t^{\mu-\nu} \varphi_{\nu \mu}(u)$. We have $\tilde{\varphi}(u) \equiv u \bmod t^{m}$. Hence $\varphi_{\nu \mu}(u)=0$ for $\mu-\nu<0$ and $\varphi_{\nu \mu}(u)=0$ for $m>\mu-\nu>0, \varphi_{\mu \mu}(u)=u$. They imply that $\varphi(u)-u \in W_{\mu-m}(\Phi(F))$. Therefore we obtain $\varphi=i d$ by (5.2.4). This completes the proof of Proposition 5.2.1 


\section{§5.5. The $G$-module structure on $\Phi(F)$}

Let $F$ be a coherent invariant sheaf and let us take $b \geq a$ such that

$$
\Phi(F)=\underset{a \leq l \leq b}{\oplus} \Phi(F)_{l} .
$$

Let us take $m>b-a$. We shall endow the structure of $G(m)$-module on $\Phi(F)$ as follows. For $g \in G(m)$, locally on $S$, there exist a morphism $f: V \rightarrow V$ such that the diagram

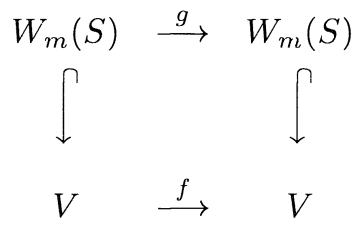

commutes. Hence $f$ is étale on a neighborhood of $i(S)$. We define the action of $g$ on $\Phi(F)=i^{*} F$ as the inverse of the composition

$$
i^{*} F_{V}=(f \circ i)^{*} F_{V} \sim i^{*} f^{*} F_{V} \stackrel{\beta(f)}{\longrightarrow} i^{*} F_{V} .
$$

This definition does not depend on the choice of $f$ by Proposition 5.2.1. This gives evidently the structure of $G(m)$-module and hence the structure of $G$ module via $G \rightarrow G(m)$. Thus we obtain the functor $\Phi$ from $I_{c}(n)$ to the category of coherent $G$-modules. Evidently $\Phi$ commutes with the tensor product.

\section{$\S 6$. The Functor $\mathcal{B}$}

\section{§6.1. Jet bundle}

Let us construct a quasi-inverse $\mathcal{B}$ of $\Phi$. We shall use a standard technique that uses jet bundles. Let us recall the definition of a jet bundle. Let $X \rightarrow T$ be a smooth morphism with fiber dimension $n$. Let $\triangle_{X / T}^{(m)}$ be the $m$-th infinitesimal neighborhood of the diagonal $X$ in $X \underset{T}{\times} X$. Let $p_{1}: \triangle_{X / T}^{(m)} \rightarrow X \underset{T}{\times} X \rightarrow X$ be the first projection and $p_{2}: \triangle_{X / T}^{(m)} \rightarrow X \underset{T}{\times} X \rightarrow X$ the second projection. The jet bundle $J_{X / T}^{(m)}$ of order $m$ is the scheme over $X$ that represents the functor

$$
X^{\prime} \mapsto\left\{\varphi ; \varphi \text { is an isomorphism from } X^{\prime} \times W^{m}\left(\mathbf{A}^{n}\right) \text { to } X_{X}^{\prime} \underset{X}{\times} \triangle_{X / T}^{(m)}\right\} .
$$

Here $X^{\prime} \underset{X}{\times} \triangle_{X / T}^{(m)}$ is the fiber product via $\triangle_{X / T}^{(m)} \stackrel{p_{1}}{\longrightarrow} X$. Hence there exists a canonical isomorphism

$$
J_{X / T}^{(m)} \times W^{m}\left(\mathbf{A}^{n}\right) \stackrel{\sim}{\longrightarrow} J_{X / T}^{(m)} \underset{X}{(m / T} \triangle_{X / T}^{(m)}
$$


Moreover the action of $G(m)$ on $W_{m}\left(\mathbb{A}^{n}\right)$ induces the action on $J_{X / T}^{(m)}$ and $\pi: J_{X / T}^{(m)} \rightarrow X$ is a principal $G(m)$ bundle. Note that $J_{X / T}^{(m)} \rightarrow X$ is locally trivial with respect to the Zariski topology of $X$.

\section{§6.2. Construction of the functor $\mathcal{B}$}

Let $M$ be a coherent $G$-module. Let us take $m \gg 0$ such that the $G$-action on $M$ comes from a $G(m)$-action on $M$.

For a morphism $X \rightarrow T$, let $\mathcal{B}(M)_{X}$ be the associated bundle of $M$ with respect to $J_{X / T}^{(m)}$. Namely let $q: J_{X / T}^{(m)} \rightarrow S$ and $\pi: J_{X / T}^{(m)} \rightarrow X$ be the projections. Then $\mathcal{B}(M)_{X}$ is the subsheaf of $\pi_{*} q^{*} M$ consisting of the sections invariant under the action of $G(m)$. Here the action of $G(m)$ on $\pi_{*} q^{*} M$ is induced by its action on $M$ and the one on $J_{X / T}^{(m)}$. This definition does not depend on $m$. In fact for $m^{\prime} \geq m$, there is a canonical $G$-equivariant morphism $J_{X / T}^{\left(m^{\prime}\right)} \rightarrow J_{X / T}^{(m)}$. Then $X \mapsto \mathcal{B}(M)_{X}$ is evidently an invariant sheaf and we shall denote it by $\mathcal{B}(M I)$. This definition does not depend on the choice of $m$ and it gives an exact functor from $\operatorname{Mod}_{c}(G)$ to $I_{c}(n)$.

\section{§6.3. $\mathcal{B}$ and $\Phi$}

We shall prove that $\mathcal{B}$ and $\Phi$ are quasi-inverse to each other. We can see easily that $\Phi \mathcal{B}(M) \cong M$ for $M \in \operatorname{Mod}_{c}(G)$. In the sequel we shall show $\mathcal{B} \Phi(F) \cong F$ for $F \in I_{c}(n)$. Let us set $M=\Phi(F)$ and let us take $b \geq a$ such that $W_{b}(M)=M$ and $W_{a-1}(M)=0$. Then for $m>b-a, G(m)$ acts on $M$. Let us take $X \rightarrow T$ in $\mathcal{S}_{n}(S)$ and let us consider the diagram

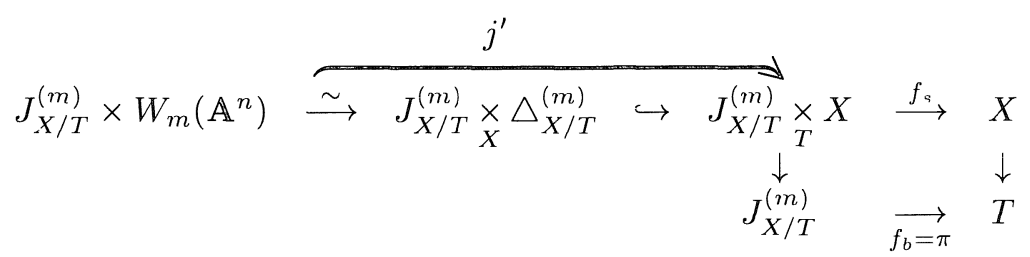

Then $\pi$ gives a morphism $f$ from $\left(J_{X / T}^{(m)} \underset{T}{\times} X \rightarrow J_{X / T}^{(m)}\right)$ to $(X \rightarrow T)$ in $\mathcal{S}_{n}(S)$ and hence an isomorphism

$$
\beta(f): f_{S}^{*} F_{X / T} \stackrel{\sim}{\longrightarrow} F_{J_{\mathrm{X} / T}^{(m)} \times X / J_{\mathrm{V} / T}^{(m)}} .
$$

Let $i: J_{X / T}^{(m)} \hookrightarrow J_{X / T}^{(m)} \times \mathbb{A}^{n}$ and $i^{\prime}: J_{X / T}^{(m)} \hookrightarrow J_{X / T}^{(m)} \times \underset{T}{X} X$ denote the embeddings. Then by Corollary 5.2.2 we have a canonical isomorphism

$$
i^{*} F_{J_{\mathrm{V} / T}^{(m)} \times \mathbf{A}^{n} / J_{X / T}^{(m)}} \simeq i^{\prime *} F_{J_{\Upsilon / T}^{(m)} \underset{T}{\times} X / J_{X / T}^{(m)}}
$$


We have $i^{*} F_{J_{\mathfrak{v} / T}^{(m)} \times \mathbf{A}^{n} / J_{\mathrm{\Upsilon} / T}^{(m)}}^{(m)}=q^{*} M$ where $q: J_{X / T}^{(m)} \rightarrow S$ is the canonical projection and $i^{\prime *} F_{J_{X / T}^{(m)} \times X}^{(m)} \times J_{\mathrm{V} / T}^{(m)}=f_{s}^{*} F_{X / T}$. We can see easily that the isomorphism $q^{*} M \simeq f_{s}^{*} F_{X / T}$ is $G(m)$-equivariant and hence $\mathcal{B}(M) \cong F_{X / T}$. This completes the proof of $\mathcal{B} \circ \Phi \cong i d$.

\section{$\S 7 . \quad$ The Weight Filtration}

We established the equivalence $\operatorname{Mod}_{c}(G)$ and $I_{c}(n)$. Since any object of $\operatorname{Mod}_{c}(G)$ has a weight filtration $W$, any object $I_{c}(n)$ has a weight filtration $W$.

The corresponding properties of $W$ for $\operatorname{Mod}_{c}(G)$ imply the following properties.

(7.1) $F \mapsto W_{l}(F)$ and $F \mapsto G r_{l}^{W}(F)$ are exact functors from $I_{c}(n)$ to $I_{c}(n)$. (7.2) For invariant sheaves $F_{1}, F_{2} \in I_{c}(n)$, we have

$$
W_{l_{1}+l_{2}}\left(W_{l_{1}}\left(F_{1}\right) \otimes W_{l_{2}}\left(F_{2}\right)\right)=W_{l_{1}}\left(F_{1}\right) \otimes W_{l_{2}}\left(F_{2}\right) .
$$

(7.3) For $F_{1}, F_{2} \in I_{c}(n)$ and $l \in \mathbb{Z}$, the above isomorphism induces an isomorphism

$$
\oplus_{l=l_{1}+l_{2}} G r_{l_{1}}^{W}\left(F_{1}\right) \otimes G r_{l_{2}}^{W}\left(F_{2}\right) \stackrel{\sim}{\longrightarrow} G r_{l}^{W}\left(F_{1} \otimes F_{2}\right) .
$$

(7.4) For $F \in I^{b}(n), W_{-l-1}\left(W_{l}(F)^{*}\right)=0$ and $G r_{l}^{W}\left(F^{*}\right) \cong\left(G r_{-l}^{W}(F)\right)^{*}$.

Thus $I^{b}(n)$ has a structure of a filtered rigid tensor category.

Example 7.1. $\mathcal{O}$ is pure of weight $0 . \Theta$ is pure of weight 1 and $\Omega^{k}$ is pure of weight $-k$.

Example 7.2. $\mathcal{P}^{(m)}$ is of weight $[-m, 0]$ (c.f. Example 1.5) and $\mathcal{P}^{(m)} / W_{-1-l}\left(\mathcal{P}^{(m)}\right)=\mathcal{P}^{(l)}$ for $0 \leq l \leq m$.

Example 7.3. $W_{m}(\mathcal{D})$ is of weight $[0, m]$ (c.f. Example 1.6) and $W_{l}\left(W_{m}(\mathcal{D})\right)=W_{l}(\mathcal{D})$ for $0 \leq l \leq m$. We have $W_{m}(\mathcal{D})=\left(\mathcal{P}^{(m)}\right)^{*}$.

\section{$\S 8 . \quad$ Lie Derivative}

\section{§8.1. Definition}

Let $F$ be a coherent invariant sheaf, $X \rightarrow T$ an object in $\mathcal{S}_{n}(S)$ and $v$ a relative tangent vector on $X / T$. Then we can define a Lie derivative $L(v)$ : 
$F_{X / T} \rightarrow F_{X / T}$ that satisfies

$$
\begin{aligned}
L(v)(a u)= & a L(v) u+v(a) u \\
& \text { for } a \in \mathcal{O}_{X} \text { and } u \in F_{X / T} .
\end{aligned}
$$

Let us set $T^{\prime}=T \times \operatorname{Spec}\left(\mathbb{Z}[\varepsilon] / \varepsilon^{2} \mathbb{Z}[\varepsilon]\right)$ and $X^{\prime}=X \times_{T} T^{\prime}$ and define an automorphism $f: X^{\prime} \rightarrow X^{\prime}$ over $T^{\prime}$ by $x \mapsto x+\varepsilon v(x)$. Let $p$ be the projection $\left(X^{\prime} \rightarrow T^{\prime}\right)$ to $(X \rightarrow T)$. Then we have a homomorphism

$$
\psi: p_{s}^{*} F_{X / T} \simeq F_{X^{\prime} / T^{\prime}} \stackrel{\beta(f)}{\longrightarrow} F_{X^{\prime} / T^{\prime}}=p_{s}^{*} F_{X / T} .
$$

Since $p_{s *} p_{s}^{*} F_{X / T}=F_{X / T} \oplus \varepsilon F_{X / T}$, we define $\psi(v)$ by $\psi(u)=u \oplus \varepsilon L(v) u$. Then $L(v)$ satisfies the relation (7.1.1). Moreover we have

$$
\begin{aligned}
{\left[L\left(v_{1}\right), L\left(v_{2}\right)\right]=} & L\left(\left[v_{1}, v_{2}\right]\right) \\
& \text { for } v_{1}, v_{2} \in \Theta_{X / T} .
\end{aligned}
$$

Note that for any $s \in F_{X / T}, v \mapsto L(v) s$ is a differential operator from $\Theta_{X / T}$ to $F_{X / T}$.

This definition coincides with the usual definition of the Lie derivative on $\Omega_{X / T}^{k}$. The Lie derivative acts on $W_{m}(\mathcal{D})$ by the adjoint action.

\section{§8.2. The infinitesimal action}

Let $\mathfrak{g}$ be the subsheaf of $p_{*}\left(\Theta_{V / S}\right)$ consisting of tangent vectors that vanishes at the zero section. Here $p: V \rightarrow S$ is the projection. Then we have

$$
\mathfrak{g}=S_{+}\left(\mathcal{V}^{*}\right) \otimes_{\mathcal{O}_{S}} \mathcal{V}
$$

where $S_{+}\left(\mathcal{V}^{*}\right)=\oplus_{l>0} S^{l}\left(\mathcal{V}^{*}\right)$. Set $W_{l}(\mathfrak{g})=\oplus_{1-l^{\prime} \leq l} S_{l^{\prime}}\left(\mathcal{V}_{*}\right) \otimes \mathcal{V}$. Then $W_{0}(\mathfrak{g})=\mathfrak{g}$ and $\mathfrak{g} / W_{-m-1}(\mathfrak{g})$ is the Lie algebra of $G(m)$. Hence for $F \in I(n), \mathfrak{g}$ acts on $\Phi(F)$ as its infinitesimal action. This action coincides with the action through the Lie derivative.

\section{§9. Characteristic Zero Case}

In this section 9, let us take $\operatorname{Spec}(k)$ as $S$ for a field $k$ of characteristic 0 . Then $V$ may be regarded as an $n$-dimensional vector space over $k$. In this case, the Lie algebra $\mathfrak{g}$ in $\S 8.2$ coincides with $S_{+}\left(V^{*}\right) \otimes V$ where $S_{+}\left(V^{*}\right)=$ $\oplus_{l>0} S^{l}\left(V^{*}\right)$. It contains the Lie algebra $V^{*} \otimes V$ of $G L(V)$. Therefore the category of $G$-modules coincides with the category of $(\mathfrak{g}, G L(V))$-modules. 
Set $W_{-l}(\mathfrak{g})=\oplus_{1-l^{\prime} \leq-l} S^{l^{\prime}}\left(V^{*}\right) \otimes V$. The action homomorphism $\mathfrak{g} \otimes M \rightarrow M$ preserves the weight filtration $W$ for a $(\mathfrak{g}, G L(V))$-module $M$. Hence if $M$ is a pure module, $W_{-1}(\mathfrak{g})$ annihilates $M$ and hence $M$ is a $G L(V)$-module. Thus we have

Proposition 9.1. Any pure invariant sheaf us semisimple.

This implies the following result by a standard argument.

Proposition 9.2. Let $F_{\nu}$ be a pure invariant sheaf of weight $w_{\nu}(\nu=$ 1,2). Then we have

$$
\operatorname{Ext}_{I^{b}(n)}^{k}\left(F_{1}, F_{2}\right)=0 \quad \text { for } \quad w_{1}-w_{2}<k .
$$

As stated in the introduction, we conjecture

Conjecture $\operatorname{Ext}_{I^{b}(n)}^{k}\left(F_{1}, F_{2}\right)=0$ for $w_{1}-w_{2} \neq k$ and $k<n$.

Since the category of $G$-modules coincides with the category of ( $\mathfrak{g}, G L(V)$ )modules, we can translate results in the Lie algebra cohomology (e.g. in $[F]$ ) in our framework. For example by the result of Goncharova $([\mathrm{G}])$, we have when $n=1$

$$
\operatorname{Ext}_{I(1)}^{l}\left(\mathcal{O}, \Omega^{1 \otimes J}\right)= \begin{cases}k & \text { for } i=0 \text { and } j=0 \\ k & \text { for } i \geq 1 \text { and } j=\left(3 i^{2}-i\right) / 2 \text { or }\left(3 i^{2}+i\right) / 2 \\ 0 & \text { otherwise }\end{cases}
$$

\section{$\S 10 . \quad$ Variants}

\section{$\S 10.1 . \quad$ Complex analytic case}

We can perform the same construction for the complex analytic case. Namely we take $\mathcal{S}_{n}$ the category of smooth morphisms $X \rightarrow T$ of fiber dimension $n$ of complex analytic spaces. A morphism $f$ from $X \stackrel{a}{\longrightarrow} T$ to $X^{\prime} \stackrel{a^{\prime}}{\longrightarrow} T^{\prime}$ is a commutative diagram

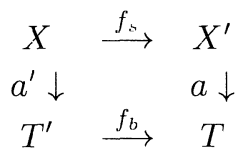

such that $X \rightarrow X^{\prime} \times_{T} T^{\prime}$ is a local isomorphism. Then the invariant sheaves are defined similarly to the algebraic case. The category of invariant sheaves (in the complex analytic case) is equivalent to the category of $G$-modules with $S=\operatorname{Spec}(\mathbf{C})$.

Hence it is equivalent to $I(n)_{\operatorname{Spec}(\mathbf{C})}$. In another word invariant sheaves are same in the complex analytic case and algebraic case. 


\section{§10.2. Multiple case}

Instead of working on the sheaves on $X$, we can work on the sheaves on $X \times_{T} X$. More precisely we can consider the following category $I(n ; 2)$. An object of $I(n ; 2)$ is the data:

(10.2.1) To any object $X \rightarrow T$ in $\mathcal{S}_{n}(S)$, assign a quasi-coherent $\mathcal{O}_{X \times_{T} X}$ modules $F_{X / T}$ whose support is contained in the diagonal set.

(10.2.2) To any morphism $\varphi=\left(\varphi_{s}, \varphi_{b}\right):(X \rightarrow T) \rightarrow\left(X^{\prime} \rightarrow T^{\prime}\right)$ in $\mathcal{S}_{n}(S)$, assign an isomorphism

$$
\beta(\varphi):\left(\varphi_{s} \times \varphi_{s}\right)^{*} F_{X^{\prime} / T^{\prime}} \stackrel{\sim}{\longrightarrow} F_{X / T} .
$$

Here $\varphi_{s} \times \varphi_{s}$ is the morphism $X^{\prime} \times_{T^{\prime}} X^{\prime} \rightarrow X \times_{T} X$ induced by $\varphi$.

We assume the similar associative law to the invariant sheaf case. We call an object of $I(n ; 2)$ a double invariant sheaf. Similarly to the invariant sheaf case we define $I_{c}(n ; 2)$ to be the category of double invariant sheaves $F$ such that $F_{X / T}$ are locally of finite presentation. For an object $X \rightarrow T$ in $\mathcal{S}_{n}(S)$, let $p_{1}: X \times_{T} X \rightarrow X$ be the projection. Then for a double invariant sheaf $F_{X / T}, X / T \mapsto p_{1 *} F_{X / T}$ is an invariant sheaf. Thus we obtain the functor

$$
p_{1 *}: I(n ; 2) \rightarrow I(n) .
$$

Let us denote by $\mathcal{O}_{\Delta^{(m)}}$ the double invariant sheaf that associates $\mathcal{O}_{\Delta_{X / T}^{(m)}}$ to $X \rightarrow T$ in $\mathcal{S}_{n}(S)$. Here $\Delta_{X / T}^{(m)}$ is the $m$-th infinitesimal neighborhood of the diagonal embedding $X \hookrightarrow X \times_{T} X$. Then for a double invariant sheaf $F$, there is an action $\mathcal{O}_{\Delta_{X / T}^{(m)}} \otimes_{\mathcal{O}_{X \times{ }_{T}{ }^{X}}} F_{X / T} \rightarrow F_{X / T}$ if we take $m$ sufficiently large. It induces $p_{1 *}\left(\mathcal{O}_{\Delta_{X / T}^{(m)}}\right) \otimes p_{1 *}\left(F_{X / T}\right) \rightarrow p_{1 *}\left(F_{X / T}\right)$. Thus we obtain a homomorphism in $I(n)$

$$
p_{1 *} \mathcal{O}_{\Delta^{(m)}} \otimes p_{1 *} F \rightarrow p_{1 *} F
$$

We can see easily

$$
\Phi\left(p_{1 *} \mathcal{O}_{\Delta(m)}\right)=p_{*} \mathcal{O}_{W^{m}(V)} .
$$

Here $p: W^{m}(V) \rightarrow S$ is the projection. We have $p_{*} \mathcal{O}_{W^{m}(V)}=S\left(\mathcal{V}^{*}\right) / W_{-m-1}$ $S\left(\mathcal{V}^{*}\right)$. Here $W_{-l}\left(S\left(\mathcal{V}^{*}\right)\right)=\oplus_{l^{\prime} \geq l} S^{l^{\prime}}\left(\mathcal{V}^{*}\right)$. Thus we obtain

Proposition 10.2.1. $I_{c}(n ; 2)$ is equivalent to a category of $G$-modules with the structure of $S\left(\mathcal{V}^{*}\right)$-modules $M$ such that $S\left(\mathcal{V}^{*}\right) \otimes M \rightarrow M$ is $G$ equivariant (more precisely $W_{-l}\left(S\left(\mathcal{V}^{*}\right)\right) M=0$ for $l \gg 0$ and $S\left(\mathcal{V}^{*}\right) / W_{-l}$ $\left(S\left(\mathcal{V}^{*}\right)\right) \otimes M \rightarrow M$ is $G$-equivariant $)$. 


\section{References}

[EGA] Grothendieck, A., Élément de Géométrie Algébrique IV, étude locale des schémas et des morphismes de schémas (Troisième partie), Inst. Hautes Études Scı. Publ. Math., 28 (1966).

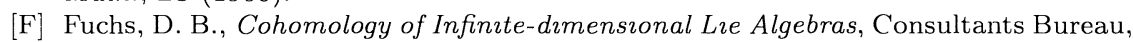
New York, A Division of Plenum Publishing Corporation, 1986.

[G] Goncharova, L. V., Cohomology of Lie algebras of formal vector fields on the line,

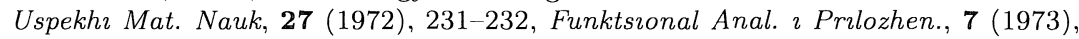
6-14. 
